\title{
Clinical Observation of rhIL-2 Combined with Zhenqi Fuzheng and BaofuKang Suppository in the Treatment of Cervical Intraepithelial Neoplasia II with HPV Infection
}

\author{
Yu Cai ${ }^{*}$, Jianjun Zhai ${ }^{1}$, Jinping Xun ${ }^{1}$, Xiaojin $\mathrm{He}^{2}$ \\ ${ }^{1}$ Department of Gynecology and Obstetrics, BeiJing TongRen Hospital, Capital Medical University, Beijing, China \\ ${ }^{2}$ Department of Pathology, BeiJing TongRen Hospital, Capital Medical University, Beijing, China \\ Email: *caiyu892002@sina.com
}

How to cite this paper: Cai, Y., Zhai, J.J., Xun, J.P. and He, X.J. (2020) Clinical Observation of rhIL-2 Combined with Zhenqi Fuzheng and BaofuKang Suppository in the Treatment of Cervical Intraepithelial Neoplasia II with HPV Infection. Open Journal of Obstetrics and Gynecology, 10, 1045-1055. https://doi.org/10.4236/ojog.2020.1080098

Received: June 30, 2020

Accepted: August 11, 2020

Published: August 14, 2020

Copyright $\odot 2020$ by author(s) and Scientific Research Publishing Inc. This work is licensed under the Creative Commons Attribution International License (CC BY 4.0).

http://creativecommons.org/licenses/by/4.0/

\begin{abstract}
Objective: To investigate the clinical effects of recombinant human interleukin-2 (rhIL-2) combined with Zhenqi Fuzheng and Baofukang on cervical intraepithelial neoplasia II (CINII) combined with human papilloma virus infection. Methods: There were 593 patients diagnosed with CINII with HPV infection, including 296 in the control group and 297 in the experimental group. The control group was given only Zhenqi Fuzheng oral and Baofukang suppository vaginal medicine. The experimental group was treated with rhIL-2 injection in addition to Zhenqi Fuzheng oral and Baofukang suppository vaginal medicine which is treated for 3 months. After 3 months, Thinprep cytologic test (TCT), human papilloma virus (HPV) quantitative examination and colposcopy biopsy were reviewed. Results: After 3 months of treatment, the negative conversion rate and total effective rate of HPV in the control group were $58.11 \%$ and $70.95 \%$ respectively, and the negative conversion rate and total effective rate of the experimental group were $79.46 \%$ and $90.57 \%$ respectively. There was significant difference between the two groups $(\mathrm{p}=0.000)$. The curative rate of cervical lesions was significantly higher in the test group than in the control group, $89.56 \%, 68.91 \%$, respectively. The statistical difference between the two groups is significant $(\mathrm{p}=0.000)$. Conclusion: It has an essential clinical value that HPV infection patients and cervical intraepithelial neoplasia II associated with HPV infection patients are treated by rhIL-2 combined with Zhenqifuzheng and Baofukang, which is safe, effective, non-invasive, reusable advantages. However, the long-term efficacy and side effects need to be further studied.
\end{abstract}




\section{Keywords}

Cervical Intraepithelial Neoplasia II, Human Papilloma Virus, Recombinant Human Interleukin-2, Zhenqi Fuzheng, Baofukang

\section{Introduction}

Cervical cancer is a serious disease threatening to human health, and many studies have demonstrated that cervical cancer and cervical intraepithelial neoplasia (CIN) (including CINI, CINII, CINIII) are infected by HPV. It is essential for persistent infection with high-risk HPV subtypes [1]. Over $80 \%$ of sexually active women would have been infected with HPV at some point by the age of 50 [2], although more than $90 \%$ of these infections are transient, and are cleared by people immune response within 6 - 18 months [3]. Even when low-grade cervical intraepithelial neoplasia (CIN1) develops, the majority of these lesions regress spontaneously back to normal, particularly in young women [4]. While they have cervical CIN, in terms of CINII, CINIII, most of them will be treated with cervical conization and physical lesion, such as cold knife conization (CKC), large loop excision of the transformation zone (LLETZ), hysteroscopic conization of cervix and laser conization and so on [5]. Moreover, postoperative complications, such as wound bleeding, infection, cervical incompetence and cervical adhesions, will affect future fertility, and the cost is high. Moreover, there is a trend of rejuvenation at present, coupled with the Beijing metropolitan area of late marriage and late childbearing, the increase in the number of Advanced Maternal Age, especially in women of reproductive age. Meta-analyses [6] [7] and large retrospective linkage studies [8] [9] suggest that the excisional methods of treatment also increase the risk of preterm birth, low birth weight, premature rupture of membranes, and perinatal mortality in subsequent pregnancies; cervical intraepithelial neoplasia itself and other confounders may contribute to that risk. [10] [11] [12]. In addition, the recurrent infection of HPV and CIN may be persistent or relapse after operation, but the cervix cannot be operated many times. Therefore, it is very crucial how to treat the HPV positive patients with CIN safely and effectively, and, how to treat relapses with low cost and easy acceptance.

In addition, the occurrence, development, therapeutic effect and prognosis of the tumor are related to the immune state of the body to some extent. There's no doubt that the improvement of the immune function of the body can inhibit the occurrence and development of tumor. Clinical and experimental data have shown that local and systemic cytokines can induce tumor regression, mainly those that could induce a cellular immune response. For patients with CIN II/III or other types of neoplasia, conservative treatment with IFN- $\alpha$ can preserve the woman's reproductive ability by avoiding the need for more invasive procedures, such as conization. Interferons (IFNs) are glycoproteins that were initially de- 
scribed with respect to their strong antiviral effects [13] [14]. A study by Ramos et al. [15] demonstrated that IFN- $\alpha$-treated patients with CIN III and with tumor regression expressed more type $1 \mathrm{~T}$ helper (Th1)-profile cytokines (IFN- $\gamma$, Tumor necrosis factor (TNF)- $\alpha$, interleukin (IL)-2), with a significant reduction in the high-risk HPV viral load in the lesions. Patients whose therapy failed were smokers and had a higher expression of Th2-type (IL-4) or regulatory T cytokines (transforming growth factor (TGF)- $\beta 2$ and TGF- $\beta 3$ ).

Interleukin- 2 is a member of the chemokine family of cytokines and is currently used in biotherapy of tumors, especially in the treatment of gastrointestinal tumors, lung cancer and cancerous pleural and peritoneal effusion. But it has been shown to promote lymphocyte growth, proliferation, differentiation, immune response and antiviral infection. In Zhenqi fuzheng capsule, the main ingredients are Astragalus, ligustrum Lucidum. It has the function of invigorating Qi, nourishing Yin and nourishing kidney, and it is mainly used for the people of deficiency of body and deficiency of Qi and Yin. It can promote the recovery of normal function with surgery, radiation and chemotherapy, but at present, Zhenqi Fuzheng is only used as adjuvant therapy to improve immunity of tumor patients. This study reviewed the use of recombinant Human Interleukin 2 combined with Zhenqi Fuzheng capsule and Baofukang suppository in patients with CINII and HPV infection diagnosed by colposcopy biopsy from January 2015 to December 2018 in the Beijing Tongren Hospital, then we study the value of them in the treatment of cervical CINII and HPV infecttion, and make effective and convenient treatment plan for cervical CINII patients with HPV infection.

\section{Materials and Methods}

\subsection{Materials}

General Data Review-Beijing Tongren Hospital from January 2015 to December 2018-Cervical smear TCT abnormal or normal, but HPV positive patients $\rightarrow$ colposcopy directed cervical biopsy $\rightarrow$ pathological report $\rightarrow$ inclusion. Inclusion criteria: 1) colposcopic biopsy was performed in patients with HPV infection; 2) all patients in the treatment group received IL-2 treatment voluntarily; 3) age of the subjects ranged from 20 - 40 years. Exclusion criteria: 1) trichomonas, mold, chlamydia, bacterial vaginosis, any one of the infections; 2) severe hypotension, severe cardiac and renal insufficiency, high fever, epilepsy; 3) pregnant or nursing women; 4) those allergic to biological agents; 5) patients with CINI or CINIII; 6) patients who had unprotected sex and couldn't be followed up on time; 7) exclusion from patients who were not followed up and change the treatment plan. Colposcopic biopsy confirmed CINII with high risk HPV infection in 600 patients according to age. Based on the principle of matching, TCT and HPV abnormalities were divided into Control group 300, Test group 300. There was no significant difference between the two groups in age and TCT $(\mathrm{P}>0.05)$. As shown in Table 1. 
Table 1. Age and TCT positive ratio in two groups.

\begin{tabular}{cccc}
\hline Group & $\mathrm{N}$ & Age $^{*}$ & TCT positive $_{(\mathrm{n} \%)^{*}}$ \\
\hline Control group & 300 & $30.9 \pm 10.1$ & $209(69.66)$ \\
Test group & 300 & $30.1 \pm 10.8$ & $215(71.67)$ \\
\hline
\end{tabular}

${ }^{*} \mathrm{P}>0.05$.

\subsection{Methods}

\subsubsection{Detection of High Risk HPV}

High risk HPV was detected by human papillomavirus PCR, the 21 human papillomavirus subtypes that can be detected include HPV16, 18, 31, 33, 35, 39, 45, $51,52,53,58,59,66,26,82,73$ and HPV6, 11, and 81. It was positive in quantitative analysis ( $>100 \mathrm{PG} / \mathrm{ml})$.

\subsubsection{Colposcopy and Cervical Biopsy}

Patients who are positive for either TCT or HPV or both take some test and pay attention to some matters, such as blood routine test, leucorrhea test, and no sexual activity or vaginal medication 48 hours before colposcopy examination. If abnormal images were found under colposcopy, biopsy was taken at the site. If no abnormal images were found, biopsy was taken at 3, 6, 9, 12 points of cervix. Meanwhile, endocervical curettage (ECC) was taken and tissue of this was sent for biopsy too.

\subsubsection{Medicine}

rhIL-2 for injection: developed and produced by Beijing Sihuan biopharmaceutical Co., Ltd., batch No.: gyzz s1097015 (1 million/0.90ml/bottle). Zhenqi Fuzheng Capsule: produced by Gansu Lanyao Pharmaceutical Co., Ltd., batch No.: gjzz62020987, 48 capsules/bottle. Take 6 capsules at a time, twice a day, orally. Baofukang suppository: produced by Hainan Bikai Pharmaceutical Co., Ltd., batch No.: gjzz46020058, 8 pieces per box.

\subsubsection{Methods}

300 people in the control group were only given Zhenqi Fuzheng oral and Baofukang suppository on Vagina. In the experimental group, 300 patients were treated with rhIL-2 in addition to Zhenqi Fuzheng oral and Baofukang suppository on Vagina.

IL-2 one million units $+2 \mathrm{ml}$ sterile water for injection, subcutaneous injection, once a day, for 2 weeks every month, as a course of treatment. After half a month interval, the next course of treatment was carried out, which is performed for 3 courses. Zhenqi Fuzheng Capsule is taken 6 capsules at a time, twice a day, orally. The course of treatment is two weeks. After half a month interval, the next course of treatment was carried out, which is performed for 3 courses. Baofukang suppository: is applied to patient by two pills to the vagina every night for 8 days in a month, which is performed for 3 courses.

\subsubsection{Observation Index}

1) General condition: observe the change of clinical symptoms and subjective 
feeling of patients before and after taking medicine, and examine the body carefully; 2) if there is any adverse reaction, deal with the symptoms; 3 ) the TCT and HPV quantitative tests were reexamined after 4 months; 4 ) a colposcopic biopsy of the person who is positive for either one or both of the re-examinations is done, and treatment is changed mid-course.

\subsubsection{Evaluation}

HPV DNA would be tested after patients had been treated for three sessions to evaluate the drugs effectiveness. Cure: HPV turns negative; Effective: HPV Ratio Drops by 2/3; Ineffective: Ratio unchanged or increased. Total effective rate is cure + effective/total\%. Negative conversion rate of HPV. Negative rate: HPV negative number/total number of cases $100 \%$, cervical lesions cure rate: cure number/total number of cases $100 \%$.

\subsection{Statistical Analysis}

SPP20.0 was used for statistical analysis, the data expressed by $(\bar{x} \pm s)$ and $t$ test was used for comparison among groups. Differences between groups of the count data were evaluated by the Chi-square test, $\mathrm{p}<0.05$. It was significant difference of $\mathrm{p}<0.05$.

\section{Results}

\subsection{Follow-Up Results}

During the follow-up period of 3 months, 3 patients had local injection pain and 2 patients had occasional low fever, which did not exceed $38^{\circ} \mathrm{C}$. In the control group, 2 cases lost follow-up and 2 cases changed treatment plan, while in the experimental group, 3 cases changed other treatment because they could not stand the local pain caused by injection.

\subsection{Comparison of HPV Negative Conversion Rate between Two Groups}

After 3 months, the negative conversion rate and total effective rate of HPV in the control group were $58.11 \%$ and $70.95 \%$ respectively, and the negative conversion rate and total effective rate of the experimental group were $79.46 \%$ and $90.57 \%$ respectively. There was significant difference between the two groups $\left(\chi^{2}\right.$ $=36.774, \mathrm{p}=0.000)$, as shown in Table 2 .

Table 2. The negative conversion rate of HPV after 4 months in the two groups (n\%).

\begin{tabular}{|c|c|c|c|c|c|}
\hline \multirow{2}{*}{ Group } & \multirow{2}{*}{$\mathrm{N}$} & \multicolumn{4}{|c|}{ HPV } \\
\hline & & Cure & Effective & Invalid & Total effective \\
\hline Control & 296 & $172(58.11)$ & $38(12.84)$ & $86(29.05)$ & $210(70.95)$ \\
\hline Test & 297 & $236(79.46)$ & 33 (11.11) & $28(9.43)$ & $269(90.57)$ \\
\hline$x^{2}$ & & & 36.774 & & \\
\hline $\mathrm{P}$ & & & 0.000 & & \\
\hline
\end{tabular}




\subsection{Comparison of Cure Rate of Cervical Lesion between Two Groups}

After 3 months of treatment, the curative rate of cervical lesions was significantly higher in the test group than in the control group, $89.56 \%, 68.91 \%$, respectively. The difference between the two groups was statistically significant $\left(\chi^{2}=\right.$ 38.430, $\mathrm{p}=0.000$ ), as shown in Table 3 .

\section{Discussion}

Cervical cancer (CC) is the third most common cancer in women worldwide accounting for $13 \%$ of all female cancers in developed countries. Persistent infection with HPV is considered the major cause of CC [16] [17] [18] [19]. Most sexually active women will have an HPV infection at some time in their life, with or without low intraepithelial lesions (LSIL), which will be spontaneously cleared by the immune system. However, in a small proportion of women the virus is able to establish a persistent infection, probably due to the synergistic effect of suboptimal host-dependent immune response and HPV-induced immunological changes, and may cause a different clinical outcome [20] [21] [22]. Escape from innate immune recognition seems to be the hallmark of HPV pathogenesis. Failure to develop an effective cell-mediated immune response results in persistent infection and increased risk of malignant transformation of the cervical cells [23] [24]. Interleukin 2 (IL-2) has been used to treat diverse types of cancer that express the IL-2 receptor (IL-2R) such as intestinal cancer [25] [26], esophageal cancer [27], and head and neck cancer [28]. The expression of IL-2R in cervical cancer cells has been reported by some groups and by our research group [29] [30]. On the other hand, IL-2R is present in normal cells of the immune system, for example, lymphocytes [31], natural killer cells [32], and dendritic cells [33]. Studies demonstrate that treatment with 100 IU of IL-2 induces a decrease in the phosphorylation of JAK3 and STAT5 proteins involved in the proliferation of cervical cancer cells [34]. In addition, the precancerous lesion of cervical cancer is cervical intraepithelial neoplasia (CIN). Its pathological diagnosis is divided into three levels, namely, CINI, II and III. 70\% - 80\% of CIN II and III can develop into carcinoma in situ and invasive cancer, so all CIN II and CINIII should be treated [35] [36] [37]; however, for young and unborn patients, the residual lesions or recovery after physical treatment or LEEP, cold knife conization and other surgical treatment are not excluded Reoperation may be the most important factor affecting the delivery. Cervical scar is not conducive to the expansion

Table 3. Cure rate of cervical lesion after 4 months in two groups.

\begin{tabular}{ccc}
\hline Group & $\mathrm{N}$ & Cure rate of cervical lesion \\
\hline Control & 296 & $204(68.91 \%)$ \\
Test & 297 & $266(89.56 \%)$ \\
$\chi^{2}$ & & 38.430 \\
$P$ & & 0.000 \\
\hline
\end{tabular}


of the uterine opening, and increases the rate of cesarean section. Moreover, the occurrence, development, therapeutic effect and prognosis of tumors are related to the immune state of the body to a certain extent. Therefore, improving the immune function of the body will undoubtedly inhibit the occurrence and development of tumors [38]. In addition, the traditional Chinese medicine believes that: the positive Qi is stored in memory, and evil cannot be done. Based on this theory, one of the basic therapeutic principles of traditional Chinese medicine is to strengthen the body's natural defense ability to achieve disease prevention and resistance. Moreover, the blood stasis memory is another important reason for tumor occurrence and metastasis, so there is a theory that it can inhibit the occurrence, development and metastasis of tumor by activating blood circulation and removing blood stasis on the basis of strengthening. So we use rhIL-2 combined with Zhenqifuzheng and Baofukang suppository to treat cervical CINII with HPV infection, and further study the best treatment plan of CINII with HPV infection.

After 3 months of treatment, the negative conversion rate and total effective rate of HPV in the control group were $58.11 \%$ and $70.95 \%$ respectively, and the negative conversion rate and total effective rate of the experimental group were $79.46 \%$ and $90.57 \%$ respectively. There was significant difference between the two groups $(\mathrm{p}=0.000)$. Moreover, after 3 months, the curative rate of cervical lesions was significantly higher in the test group than in the control group, $89.56 \%, 68.91 \%$, respectively. The statistical difference between the two groups is significant $(\mathrm{p}=0.000)$.

The results showed that rhIL-2 combined with Zhenqifuzheng and Baofukang suppository was superior to Zhenqifuzheng and Baofukang suppository in the treatment of cervical CINII combined with HPV infection. However, this is slightly lower than the therapeutic effect of rhIL-2 and cervical circumcision in the treatment of CINI combined with HPV infection [39] [40]. However, this treatment plan avoids the invasive treatment of LEEP, the abortion and premature delivery caused by cervical dysfunction after operation, or the difficulty of delivery caused by cervical scar formation, which increases the chance of cesarean section. In addition, it is also effective for recurrent patients and patients with persistent HPV infection. Only immunotherapy alone can cure most of the patients with HPV infection and CINII combined with HPV infection. The reasons may be as follows: 1) rhIL-2 is a kind of lymphokine, which can induce cytotoxic T cells, natural killer cells and lymphokine activated killer cells (LAK) to increase in value, enhance its killing activity, and then clear the tumor cells in vivo, also can enhance the secretion of antibody and interferon, with the functions of anti-tumor, anti-virus infection, antibacterial infection and improving immunity; 2) It is the core substance of the immune response of the body, and it is an immunomodulator [41] [42]; 3) Zhenqi Fuzheng can significantly increase $\mathrm{CD} 3+, \mathrm{CD} 4+\mathrm{T}, \mathrm{CD} 4+/ \mathrm{CD} 3+$ ratio, enhance the activity of NK cells, improve the cellular and humoral immune functions of the body, and play an anti-tumor role [43]. 


\section{Conclusion}

In a word, it has an essential clinical value that cervical intraepithelial neoplasia II associated with HPV infection patients are treated by rhIL-2 combined with Zhenqifuzheng and Baofukang, which is safe, effective, non-invasive, reusable advantages. However, the long-term efficacy and side effects need to be further studied. Moreover, the reason why the therapeutic effect is not more than $90 \%$, has to be further analyzed, whether it is related to age, sexual partner, condom use and menstruation and so on. In addition, during the outbreak of the novel coronavirus, in order to cut down hospital admissions, decrease the number of patients coming back to the hospital, and reduce patient aggregation and cross-infection, patients were performed a colposcopy biopsy in outpatient, and were prescribed drugs for treatment of cervical lesions for three months based on the colposcopy results, and have achieved satisfactory results.

\section{Acknowledgements}

He Xiao-jin teacher in department of pathology provided technical support.

\section{Conflicts of Interest}

The authors declare no conflicts of interest regarding the publication of this paper.

\section{References}

[1] Boulet, G.B., Horvath, C., Broeck, D.V., Sahebali, S. and Borgers, J. (2007) Human Papillomavirus: E6 and E7 Oncogenes. The International Journal of Biochemistry \& Cell Biology, 39, 2006-2011. https://doi.org/10.1016/j.biocel.2007.07.004

[2] Moscicki, A.B. (2005) Human Papillo Mavirus, Papanicolaou Smears, and the College Female. Pediatric Clinics of North America, 52, 163-177. https://doi.org/10.1016/j.pcl.2004.10.005

[3] Plummer, M., et al. (2007) A 2-Year Prospective Study of Human Papillomavirus Persistence among Women with a Cytological Diagnosis of Atypical Squamous Cells of Undetermined Significance or Low-Grade Squamous Intraepithelial Lesion. The Journal of Infectious Diseases, 195, 1582-1589. https://doi.org/10.1086/516784

[4] Jordon, J., et al. (2008) European Guidelines for Quality Assurance in Cervical Cancer Screening: Recommendations for Clinical Management of Abnormal Cervical Cytology, Part 1. Cytopathology, 19, 342-354. https://doi.org/10.1111/j.1365-2303.2008.00623.x

[5] Diane, E. Halliwell, M.K., Anita, M., Ilkka, K., Evangelos, P., Georgios, Th., et al. (2016) Tracking the Impact of Excisional Cervical Treatment on the Cervix Using Biospectroscopy. Scientific Reports, 6, Article No. 38921. https://doi.org/10.1038/srep38921

[6] Castañon, A., Landy, R., Brocklehurst, P., et al. (2015) Is the Increased Risk of Preterm Birth Following Excision for Cervical Intraepithelial Neoplasia Restricted to the First Birth Post Treatment? BJOG, 122, 1191-1199. https://doi.org/10.1111/1471-0528.13398

[7] Bruinsma, F.J. and Quinn, M.A. (2011) The Risk of Preterm Birth Following Treat- 
ment for Precancerous Changes in the Cervix: A Systematic Review and Meta-Analysis. BJOG, 118, 1031-1041. https://doi.org/10.1111/j.1471-0528.2011.02944.x

[8] Albrechtsen, S., Rasmussen, S., Thoresen, S., Irgens, L.M. and Iversen, O.E. (2008) Pregnancy Outcome in Women before and after Cervical Conisation: Population Based Cohort Study. BMJ, 337, a1343. https://doi.org/10.1136/bmj.a1343

[9] Noehr, B., Jensen, A., Frederiksen, K., Tabor, A. and Kjaer, S.K. (2009) Depth of Cervical Cone Removed by Loop Electrosurgical Excision Procedure and Subsequent Risk of Spontaneous Preterm Delivery. Obstetrics \& Gynecology, 114, 1232-1238. https://doi.org/10.1097/AOG.0b013e3181bf1ef2

[10] Castanon, A., Brocklehurst, P., Evans, H., Peebles, D., Singh, N., Walker, P., et al. (2012) Risk of Preterm Birth after Treatment for Cervical Intraepithelial Neoplasia among Women Attending Colposcopy in England: Retrospective-Prospective Cohort Study. BMJ, 345, e5174. https://doi.org/10.1136/bmj.e5174

[11] Kyrgiou, M., Arbyn, M., Martin-Hirsch, P. and Paraskevaidis, E. (2012) Increased Risk of Preterm Birth after Treatment for CIN. BMJ, 345, e5847. https://doi.org/10.1136/bmj.e5847

[12] Bruinsma, F., Lumley, J., Tan, J. and Quinn, M. (2007) Precancerous Changes in the Cervix and Risk of Subsequent Preterm Birth. British Journal of Obstetrics and Gynaecology, 114, 70-80. https://doi.org/10.1111/j.1471-0528.2006.01107.x

[13] Friedman, R.M. (1967) Interferon Binding: The First Step in Establishment of Antiviral Activity. Science, 156, 1760-1761. https://doi.org/10.1126/science.156.3783.1760

[14] Michelin, M.A., Montes, L., Nomelini, R.S., Trovó, M.A. and Murta, E.F.C. (2015) Helper T Lymphocyte Response in the Peripheral Blood of Patients with Intraepithelial Neoplasia Submitted to Immunotherapy with Pegylated Interferon- $\alpha$. International Journal of Molecular Sciences, 16, 5497-5509. https://doi.org/10.3390/ijms16035497

[15] Ramos, M.C., Mardegan, M.C., Peghini, B.C., Adad, S.J., Michelin, M.A. and Murta, E.F.C. (2010) Expression of Cytokines in Cervical Stroma in Patients with High-Grade Cervical Intraepithelial Neoplasia after Treatment with Intralesional Interferon $\alpha$-2b. European Journal of Gynaecological Oncology, 31, 522-529.

[16] Arbyn, M., Fabri, V., Temmerman, M. and Simoens, C. (2014) Attendance at Cervical Cancer Screening and Use of Diagnostic and Therapeutic Procedures on the Uterine Cervix Assessed from Individual Health Insurance Data (Belgium, 2002-2006). PLoS ONE, 9, e92615. https://doi.org/10.1371/journal.pone.0092615

[17] Bosch, F.X., Broker, T.R., Forman, D., Moscicki, A.B., Gillison, M.L., Doorbar, J., et al. (2013) Comprehensive Control of Human Papillomavirus Infections and Related Diseases. Vaccine, 31, I1-I31. https://doi.org/10.1016/j.vaccine.2013.07.026

[18] Munoz, N. (2000) Human Papillomavirus and Cancer: The Epidemiology Evidence. Journal of Clinical Virology, 19, 1-5. https://doi.org/10.1016/S1386-6532(00)00125-6

[19] Walboomers, J.M., Jacobs, M.V., Manos, M.M., Bosch, F.X., Kummer, J.A., Shah, K.V., et al. (1999) Human Papillomavirus Is a Necessary Cause of Invasive Cervical Cancer Worldwide. The Journal of Pathology, 189, 12-19. https://doi.org/10.1002/(SICI)1096-9896(199909)189:1<12::AID-PATH431>3.0.CO; $\underline{2-\mathrm{F}}$

[20] Bosch, F.X., Burchell, A.N., Schiffman, M., Giuliano, A.R., de Sanjose, S., Bruni, L., et al. (2008) Epidemiology and Natural History of Human Papillomavirus Infec- 
tions and Type-Specific Implications in Cervical Neoplasia. Vaccine, 26, K1-K16. https://doi.org/10.1016/j.vaccine.2008.05.064

[21] Hubert, P., Caberg, J.H., Gilles, C., Bousarghin, L., Franzen-Detrooz, E., Boniver, J., et al. (2005) E-Cadherin-Dependent Adhesion of Dendritic and Langerhans Cells to Keratinocytes Is Defective in Cervical Human Papillomavirus-Associated (Pre)Neoplastic Lesions. The Journal of Pathology, 206, 346-355. https://doi.org/10.1002/path.1771

[22] Rositch, A.F., Koshiol, J., Hudgens, M.G., Razzaghi, H., Backes, D.M., Pimenta, J.M., et al. (2013) Patterns of Persistent Genital Human Papillomavirus Infection among Women Worldwide: A Literature Review and Meta-Analysis. International Journal of Cancer, 133, 1271-1285. https://doi.org/10.1002/ijc.27828

[23] Stanley, M.A. (2012) Epithelial Cell Responses to Infection with Human Papillomavirus. Clinical Microbiology Reviews, 25, 215-222.

https://doi.org/10.1128/CMR.05028-11

[24] Tommasino, M. (2013) The Human Papillomavirus Family and Its Role in Carcinogenesis. Seminars in Cancer Biology, 26, 13-21.

https://doi.org/10.1016/j.semcancer.2013.11.002

[25] Reinecker, H.C. and Podolsky, D.K. (1995) Human Intestinal Epithelial Cells Express Functional Cytokine Receptors Sharing the Common Gamma C Chain of the Interleukin 2 Receptor. Proceedings of the National Academy of Sciences of the United States of America, 92, 8353-8357. https://doi.org/10.1073/pnas.92.18.8353

[26] Ciacci, C., Mahida, Y.R., Dignass, A., Koizumi, M. and Podolsky, D.K. (1993) Functional Interleukin-2 Receptors on Intestinal Epithelial Cells. Journal of Clinical Investigation, 92, 527-532. https://doi.org/10.1172/JCI116598

[27] Wang, L.S., Chow, K.C., Li, W.Y., Liu, C.C., Wu, Y.C. and Huang, M.H. (2000) Clinical Significance of Serum Soluble Interleukin 2 Receptor-Alpha in Esophageal Squamous Cell Carcinoma. Clinical Cancer Research, 6, 1445-1451.

[28] Saito, T., Yoda, J. and Tabata, T. (1991) Local Administration Trials of Interleukin-2 for Head and Neck Cancer. Auris Nasus Larynx, 18, 169-178. https://doi.org/10.1016/S0385-8146(12)80221-5

[29] Rocha, L., Huitron, C., Caceres, J.R., et al. (2004) Interleukin-2 (IL-2) Receptor- $\beta \gamma$ Signaling Is Activated by c-Kit in the Absence of IL-2, or By Exogenous IL-2 via JAK3/STAT5 in Human Papillomavirus-Associated Cervical Cancer. Cellular Signalling, 16, 1239-1247. https://doi.org/10.1016/j.cellsig.2004.03.011

[30] Rangel, R., Corona, T., Soto, I., et al. (2010) Evidence That Cervical Cancer Cells Secrete IL-2, Which Becomes an Autocrine Growth Factor. Cytokine, 50, 273-277. https://doi.org/10.1016/j.cyto.2010.02.013

[31] Rosenberg, S.A., Yannelli, J.R., Yang, J.C., et al. (1994) Treatment of Patients with Metastatic Melanoma with Autologous Tumor-Infiltrating Lymphocytes and Interleukin 2. Journal of the National Cancer Institute, 86, 1159-1166. https://doi.org/10.1093/jnci/86.15.1159

[32] Lazarenko, L.M., Nikitina, O.E., Nikitin, E.V., Demchenko, O.M., Kovtonyuk, G.V., Ganova, L.O., et al. (2014) Development of Biomarker Panel to Predict, Prevent and Create Treatments Tailored to the Persons with Human Papillomavirus-Induced Cervical Precancerous Lesions. EPMA Journal, 5, 1878-5085. https://doi.org/10.1186/1878-5085-5-1

[33] Granucci, F., Zanoni, I., Feau, S. and Ricciardi, C.P. (2003) Dendritic Cell Regulation of Immune Responses: A New Role for Interleukin 2 at the Intersection of Innate and Adaptive Immunity. The EMBO Journal, 22, 2546-2551. 
https://doi.org/10.1093/emboj/cdg261

[34] Valle, A., Weiss, B., Rocha, L. and Soto, I. (2014) IL-2 Enhances Cervical Cancer Cells Proliferation and JAK3/STAT5 Phosphorylation at Low Doses, While at High Doses IL-2 Has Opposite Effects. Cancer Investigation, 32, 115-125. https://doi.org/10.3109/07357907.2014.883526

[35] Elit, L., Levine, M.N., Julian, J.A., et al. (2011) Expectant Management versus Immediate Treatment for Low-Grade Cervical Intraepithelial Neoplasia. Cancer, 117, 1438-1445. https://doi.org/10.1002/cncr.25635

[36] Bansal, N., Wright, J.D., Cohen, C.J., et al. (2008) Natural History of Established Low Grade Cervical Intraepithelial (CIN1) Lesions. Anticancer Research, 28, 1763-1766.

[37] Castle, P.E., Gage, J.C., Wheeler, C.M., et al. (2011) The Clinical Meaning of a Cervical Intraepithelial Neoplasia Grade 1 Biopsy. Obstetrics \& Gynecology, 118, 1222-1229. https://doi.org/10.1097/AOG.0b013e318237caf4

[38] Lee, J.C., Lee, K.M., Kim, D.W., et al. (2004) Elevated TGF- $\beta 1$ Secretion and Down-Modulation of NKG2D Underlies Impaired NK Cytotoxicity in Cancer Patients. The Journal of Immunology, 172, 7335-7340. https://doi.org/10.4049/jimmunol.172.12.7335

[39] Cai, Y., Zhai, J.-J., Duan, X.-Z., He, C. and Tian, X.-L. (2017) Clinical Effect of Recombinant Human Interleukin-2 and Cervical Loop Electrosurgical Excision Procedure for Cervical Intraepithelial Neoplasia I Complicated with Human Papillomavirus Infection: A Comparative Study. Guangxi Medical Journal, 39, 1654-1656.

[40] Cai, Y., Zhai, J.-J. and Duan, X.-Z. (2018) Clinical Effect of Recombinant Human Interleukin-2 Combined with BaofuKang Suppository in Patients with Cervical HPV Infection. Guangxi Medical Journal, 40, 2666-2671.

[41] Lagunas-Cruz, M.C., Valle-Mendiola, A., Trejo-Huerta, J., Rocha-Zavaleta, L., Mora-García, M.L., Gutiérrez-Hoya, A., et al. (2019) IL-2 Induces Transient Arrest in the G1 Phase to Protect Cervical Cancer Cells from Entering Apoptosis. Journal of Oncology, 2019, Article ID: 7475295. https://doi.org/10.1155/2019/7475295

[42] Hussain, S.K., Madeleine, M.M., Johnson, L.G., Du, Q., Malkki, M., Wilkerson, H.-W., et al. (2008) Cervical and Vulvar Cancer Risk in Relation to Joint Effects of Cigarette Smoking and Genetic Variation in Interleukin 2. Cancer Epidemiology, Biomarkers \& Prevention, 17, 1790-1799. https://doi.org/10.1158/1055-9965.EPI-07-2753

[43] Hu, S.-M., Dong, H., Tu, S.H., Wang, K.-F., He, B.-S. and Yang, J.-X. (2005) Clinical Study on Effect of Zhenqi Fuzheng Granule Combined with Chemotherapy in Treating Gastric Cancer. China Pharmacist, 8, 761-763. 